\title{
"Focus on the Good" - Participant Perspectives after Engaging in an Online Pain Self-Management Program
}

\author{
Marian Wilson, PhD, MPH, RN \\ Washington State University \\ College of Nursing \\ marian.wilson@wsu.edu
}

\author{
Michele R. Shaw, PhD, RN \\ Washington State University \\ College of Nursing \\ michele_shaw@wsu.edu
}

\begin{abstract}
The purpose of this study was to evaluate participants' perspectives after engaging in an 8week online pain self-management program. Unexplored qualitative data for this analysis were collected via secure online surveys distributed during a previously published randomized controlled trial. Participants for the present study were 55 adults prescribed opioid medicines for chronic pain. A qualitative descriptive approach using content analysis methods was used to analyze data obtained from three open-ended survey questions. Five common themes were identified from participant responses regarding online participation. Three themes described the benefits of the program and included: positive reframing, improved accountability, and feeling supported. Two themes described how participants would like to improve the program experience and included: desire for personalizing and ease of use. Participants' insights regarding perceived benefits and limitations can assist health care providers in understanding how online programs may assist in chronic disease selfmanagement for a multitude of health problems.
\end{abstract}

\section{Introduction}

Innovations are needed to provide people with chronic pain access to evidence-based options for managing symptoms. Cognitive and behavioral therapies that are known to assist pain control are not always accessible due to costs, insurance reimbursement structures, and stigma and receptivity related to psychological care [1]. Online programs show promise in delivering cognitive behavioral therapy (CBT) approaches that teach people to recognize relationships between thoughts, feelings and behaviors. Unfortunately, CBT has been underutilized and participants with pain do not always engage to the fullest extent, thereby lessening the effectiveness [2,3]. Limited qualitative research has been conducted to better understand participants' experiences and improve online pain selfmanagement program delivery. Therefore, this qualitative study was nested within a randomized controlled trial (RCT) that was previously published. The prior study reported on the quantitative effects for participants engaged in the online "Chronic Pain Management Program" [3]. The present study builds on what was previously learned using content analysis methods to examine unexplored data regarding participants' perspectives. Such information is necessary to provide insight on program benefits and limitations in order to maximize the usefulness of online health programs.

\section{Background}

An estimated 25 million (11\%) United States (U.S.) adults experience chronic pain, defined as any pain lasting $\geq 3$ months that does not respond to treatment [4]. More than half of those with chronic pain describe it as "unbearable" or "excruciating" [5]. Behavioral and cognitive therapies have been wellestablished in research literature as effective components that can improve pain treatment outcomes [6,7]. Yet, treatment-seeking adults with chronic pain are most likely to receive pharmacological interventions and less likely to access psychologically-based treatments [1]. Un-fortunately, a reliance on a biomedical approach to pain care in the U.S. has resulted in a $600 \%$ rise in opioid prescriptions over the past decade [1]. Concurrently, deaths caused by opioid overdose increased $300 \%$ [8]. The majority of deaths occur as an unintended consequence of legitimate prescribing practices [9]. Respiratory depression is the main hazard of opioid use [10]. It can occur as a result of opioid tolerance that develops over time as more opioids are required to receive the same pain-relieving results [10]. As 
scrutiny increases towards prescribing physicians and patients who receive opioids, a critical need exists to offer effective, affordable multidisciplinary treatment approaches that can be widely disseminated.

The U.S. Department of Health and Human Services' National Pain Strategy calls for better access to quality pain care that does not rely solely on medication [11]. Non-opioid and non-pharmacologic treatments are now recommended as the preferred treatment for chronic pain [12,13]. It has been proposed that the call to reduce opioid prescriptions may be achieved by increasing emphasis on nonpharmacologic treatments [14]. Having accessible, affordable options is key to reducing opioid use and the associated risks for people with chronic pain.

\subsection{Self-management interventions}

Self-management programs are intended to assist people in mastering the tasks needed to live with a chronic condition. Such programs aim to increase confidence, or self-efficacy, in one's ability to manage health symptoms [15]. Pain self-management interventions have been recommended as an essential component of evidence-based clinical practice guidelines for chronic pain [16]. Online and face-toface self-management interventions have demonstrated improved outcomes in small, specific populations of patients who suffer with a variety of painful conditions, such as patients with fibromyalgia, headaches, and arthritis [15,6]. However, no such interventions have been accepted for widespread use in the general population of patients with chronic pain, and the optimal means to engage patients in pain self-management strategies remains unclear. Specifically, for those people who require opioid medicines for pain relief, more information is needed about how best to engage them in self-management programs and maximize desired effects [3].

E-health can deliver health resources or health care via electronic means. E-health offers one possible means of access to self-management for people with chronic pain, who are increasingly using the Internet for information and support [17]. The Ehealth program used in the present study, the Chronic Pain Management Program (CPMP), was created by psychologists who are pain researchers. The CPMP is available to the public online with a paid subscription (approximately \$25 U.S. dollars per month). The CPMP is a self-directed, self-paced Internet-based self-management program intended for a general population of people with persistent non-cancer pain. The program targets cognitive, emotional, behavioral and social pain determinants. The main lessons provided map onto four modules that can generally be completed across 8-weeks and include: Thinking Better, Feeling Better, Doing More, and Relating Better. More description is provided on the program website https://pain.goalistics.com [18]. Learning modules include didactic materials and interactive activities. For example, the Thinking Better module asks participants to recognize, stop, evaluate, and redirect their self-defeating thoughts, using the same techniques a cognitive behavioral therapist might teach clients. Feeling Better guides participants through relaxation exercises and builds awareness of emotional triggers. Doing More teaches pacing and fitness activities. Relating Better assists in building a helpful support system and scheduling social activities. At the end of each activity, participants are asked to assign a helpfulness rating using a 1-to-5star rating where 1 star $=$ "not at all helpful" and 5 stars = "extremely helpful." Some activities are to be completed off-line, such as physical exercises, relaxation, or self-monitoring behaviors.

Prior research demonstrated the CPMP's ability to decrease pain severity, pain-related interference, perceived disability, depression, and pain-induced fear among participants recruited from the Internet [18]. At the time of recruitment for our RCT in 2012, the CPMP was the only online self-management program specifically developed for chronic pain that was found to be publicly available. That trial randomized 114 people with chronic non-cancer pain who had a current opioid prescription into treatment and control groups. The main published findings were that $20.9 \%$ of CPMP users compared to $6.8 \%$ of control group participants reported decreasing or discontinuing their opioid medication [3]. Moreover, in the CPMP, relative to control, participants reported significantly greater decreases in opioid misuse, increases in pain self-efficacy, and a significantly greater proportion had a clinically significant decrease (i.e. $\geq 2$ points) in pain intensity (18\% vs. $6 \%$ ) [3]. Thus, the E-health program was found to improve several outcomes valued by providers and patients. However, the improvements were modest and a large number of participants engaged minimally with the program activities. Those who engaged the most had the greatest reductions in symptoms of pain intensity and pain interference, and the largest gains in pain self-efficacy [3]. Therefore, the present qualitative descriptive study was conducted in hopes of discovering clues that could assist program engagement and result in improved efficacy.

E-health is thought to be advantageous, particularly considering the stigmatization of those with chronic pain who seek opioid treatment. Yet, a systematic review of online pain self-management 
programs found study withdrawal rates ranging from to $6 \%$ to $59 \%$ [19]. Level of distressing symptoms (either higher or lower levels) and younger age were associated with withdrawing from studies, which provides some information about why people may or may not fully engage in pain self-management programs or studies. A deeper analysis into the participant experience can inform enhancements to existing programs and identify optimal delivery methods for lasting, desired effects.

\section{Methods}

Qualitative descriptive methodology and qualitative content analysis methods were selected in order to describe participants' experiences with the online pain management program and to identify common themes [20,21]. Qualitative description is used when the goal of the research is to summarize descriptions of events or experiences in a way that depicts the perspectives of the participants [20,21]. Common themes are identified in qualitative description to provide definitions and details of the most prominent ideas provided by the participants' responses [20,21]. The methodology compliments the purpose of the present study by allowing the participants of the online pain self-management program to give subjective input about the programs' usefulness. Our previously published RCT did not include qualitative analysis and the researchers believed the additional qualitative approach used in this follow-up study would provide rich detail from the participants' unique perspectives.

Data were previously collected from U.S. adult participants $(n=55)$ prescribed opioid therapy for chronic pain who were enrolled in the RCT and engaged in the selected online self-management program, the CPMP. Results of that trial are reported elsewhere [3]. A total of 236 potential participants were screened for the original study, and 24 were found to be ineligible, primarily due to no Internet access $(33 \%)$ or planned surgery $(29 \%)$. A total of 114 participants consented to join the original RCT and 92 completed all study procedures $(19.3 \%$ attrition). Sixty (53\%) were referred from their health care provider and $54(47 \%)$ were self-referred from Internet advertising on web pages for pain (e.g., Facebook). The present study sample of 55 is comprised of the original treatment group participants $(n=45)$ plus the subsample of 8 participants who chose to trial the CPMP after serving in the control group. Notably, the original RCT exclusively recruited people prescribed opioids, unlike all other known Internet-based pain self- management studies at that time. The participant follow-up was, therefore, limited in duration to 8weeks to allow participants sufficient time to engage fully in the CPMP, and also to address questions related to study feasibility, participant engagement, and retention prior to investing resources in a longer trial.

Eligibility criteria included individuals 18 years of age or older who: (1) self-identified as having had a non-cancer chronic pain lasting for greater than 3 months; (2) had current prescription(s) for opioid medicine; (3) had Internet access with email capability either at home or at a public setting; and (4) had ability to read, speak and write in the English language. Exclusion criteria were chosen to limit confounding treatment effects and included: (1) planned surgical treatment in the next 2 months; (2) pregnancy; and/or (3) currently enrolled in therapy or support group with counselor, psychologist or psychiatrist for chronic pain or substance abuse.

All procedures were reviewed and approved by the Institutional Review Board of the university sponsoring the study. Surveys were collected online using a secure survey site. Pertinent to the qualitative descriptive study presented here were the open-ended survey questions included at the end of the study to evaluate participants' experiences with using the online pain self-management program. The three questions used for our present analysis were: 1) "Did you find anything about this program especially useful? What would that be?" 2) "Is there anything you would change about this program if you could? What would that be?" and 3) "What else can you share about your experience participating in this program?" The text responses to the open-ended questions provided the data for this qualitative descriptive study.

Ryan and Sawin's Individual and Family SelfManagement Theory (IFSMT) (2009) provided theoretical background to our study [22]. It is a descriptive, mid-level theory that allows researchers to incorporate the complexity of the human experience and build on what is known about selfmanagement [22]. In the IFSMT, the individual or family assumes responsibility for self-management, and may include health care providers as collaborators. Using the IFSMT as a guide, we can explore participants' experiences and consider unique physical, social and individual variables that may enhance or deter from achieving desired selfmanagement program outcomes [22]. 


\section{Data Analysis}

Qualitative content analysis methods as described by Schreier were used to analyze the data [23]. The data (responses to the three open-ended survey questions) were de-identified and transferred into a word document table for analysis. In order to meet the purpose of the present study, throughout the content analysis the researchers focused on identifying common themes in the text that were associated with participants' descriptions of the benefits and challenges of completing the online program [23]. Qualitative content analysis included combing concept-driven and data-driven analysis approaches to the text [23]. This means that the research team used their content expertise, qualitative expertise, prior experiences, and commonalities within the data to identify themes [23]. Following Schreier's qualitative content analysis methods, the authors initially read through the word document of the participants' responses separately and made notes describing their ideas for potential themes based on commonly identified statements throughout the data. The researchers then met in person to discuss and compare initial findings. This meeting included reviewing initial summaries of overall impressions of the data, identifying agreed upon themes, comparing supporting quotes, and then naming and describing each theme [23]. During this initial meeting, a coding frame was developed that included the agreed upon themes [23]. The researchers returned back to the data and using the coding frame as a reference, continued on with analysis by further summarizing themes, continuing coding of data, along with contrasting similarities and differences among themes [23]. The researchers met several more times throughout this process to compare individual analyses, revise themes and definitions, and compare identified quotes supporting the themes [23]. Reliability of the study was addressed by the process of having each researcher initially review and analyze the data prior to each meeting and then compare consistency of agreement between the coders [23]. Consistency was high among the commonly identified themes and supporting quotes. Validity was also addressed by considering the applicability of the themes when compared to the participants' responses and the overall purpose of the study [23]. An audit trail was kept throughout the analysis process to document decisions and next steps. Quotations from the respondents were used to support the claims made.

\section{Findings}

Five common themes were identified that provide description of the participants' perspectives about the online program. Themes describing the benefits of the program included: (1) positive reframing, (2) improved accountability, and (3) feeling supported. Themes describing program challenges and ideas for how participants would like to improve the online program experience included: (4) desire for personalizing and (5) ease of use. Participants were predominantly women $(80 \%)$, average age 47 years (SD 10.4) and 78\% reported having education levels higher than a high school diploma. Most participants (73\%) had more than one medical diagnosis related to their painful condition with the most common being back or spine pain conditions (45\%), fibromyalgia (29\%), arthritis (26\%), or migraine headache $(22 \%)$.

\subsection{Positive reframing}

The participants commonly reported that the online pain program provided new information and techniques that could be used to make positive shifts in the way they perceive pain. Many discussed how new coping skills were gained by participating in the program and that this helped them change their negative responses and thoughts into a more positive perspective. Positive reframing as a benefit of the program can be illustrated by the following quotes:

It was extremely helpful for me to shift my mind and spirit to focus on the good and wellness instead of sickness! Huge change in me personally!

I thought that changing thinking patterns is a very important component, since chronic pain can entrench negative thought patterns.

Participants shared comments about how the program provided them with new tools to be able to change the way they approached dealing with their chronic pain. They were better able to alter their responses to the pain and attempted to see their situations in a different light, as illustrated by the following comments:

This program really helped me to realize that chronic pain and a diagnosis of a condition that has no cure...doesn't mean it's hopeless. I've come to realize that a lot of how I need to deal with the pain is my attitude. 
It's good for keeping myself feeling better about my pain because I have more tools for dealing with it.

Participants frequently shared comments about noticing improvements in their coping skills when living with their chronic pain and this allowed them to "reframe" the way they thought about pain. They acknowledged that the pain was still there but they were better able to live with it:

The most helpful part of the program for me was the attitude adjustment such as not being so hard on myself.

My actual pain is about the same, but it seems to cause me less stress. I feel I have better coping mechanisms in place now.

\subsection{Improved accountability}

The online program provided frequent reminders which helped participants improve accountability of their actions. Participants were able to identify their unique needs and take responsibility for meeting them. The program's built-in reminders cued participants to check in with their thoughts and actions. The theme of improved accountability also included participants' continuous self-evaluation of progress and steps taken to reach goals. Participants shared these examples:

It was almost like having someone ask me not to think so negatively several times a day. That alone was very helpful, at least to me.

I've found that being more self-aware is very helpful, focusing on what your body and mental state is doing to your overall pain.

Receiving reminders was described frequently as a benefit of the program that encouraged participants to continue to use the new skills they were learning. The organization that the online program provided was helpful to participants in that they found having a set plan improved coping with their pain. Benefits of improved accountability are described by the following two participants:

It created structure for my days/weeks which really helped me deal with the exhaustion and stress that goes along with the chronic pain.

I loved the idea of getting reminders on my cell phone every two hours to check my thoughts, and have texts include positive affirmations.

Completing the online pain self-management program encouraged participants to evaluate their thoughts and their overall health status. Improved accountability led to improvements in living with chronic pain, in one participant's words:

I am back on track. Getting regular newsletters, reminders, encouragement is a necessity for me.

\subsection{Feeling supported}

The theme of feeling supported was described as participants decreased feelings of isolation and being misunderstood. Participants of the online program reported gaining support and understanding from their peers who shared similar stories of living with chronic pain as illustrated in the following examples:

I liked the chat groups - it really makes you aware you're not alone and other people understand.

I really enjoy being able to share my pain and experiences with others, it really helps knowing you're not alone.

Before, I felt so alone that no one understood me.

The benefit of feeling like a part of a group was often described as a reward of the program. Participants reported a realization that there are many others who are also living with chronic pain. This sense of sharing others' experiences was an expressed benefit of the support received as part of the program.

\subsection{Desire for personalizing}

The desire for more personalizing of the online program was commonly described in the data. Participants expressed the need to be able to provide further explanation or more of their personal story than what the online program allowed them to do. Participants shared ideas for how to improve the online program as illustrated in the following:

Sometimes the answer is not always yes or no without explanation. I realize that's not necessarily a part of the "program" but at times I would like to explain how I feel. 
To better serve others like myself who have already been exposed to many of these concepts, it would help to have a way to personalize things.

Participants shared how they wanted more options in the program to meet individual needs and to be able to provide more in-depth information. One participant's quote illustrates this clearly:

I'm open to making changes, but felt the way the program was set up, that there was no way to start from where I was at for modifications. The program seemed to assume I was starting from nothing. In reality, I have a solid pain management routine in place that includes all components the program is introducing.

Taking into account the context of each participant's unique background and experiences was voiced as a perceived challenge of the online pain management program. Improving the amount of personalization was a suggested way to improve involvement.

\subsection{Ease of use}

Ease of use of the online program was another commonly identified challenge. Participants desired more clarity with the directions, reduced effort of navigation, and more orientation early on explaining how to best use the online program. Experiencing technical difficulties while using the online program was reported by some. Ease of use issues are expressed by the following participants, along with specific improvement suggestions:

A fuller, detailed explanation would have had me fully engaged right from the start. For people who aren't especially computer savvy, like me, it took a while to figure things out.

I think I need a tutorial. I did want to do the program and sort of failed at it.

Frustration at not being able to fully utilize all aspects of the online program was shared by several participants. Others found the online program complicated to navigate and/or requiring too much effort as seen in these examples:

I found it very hard to go back every day and check in and keep up with check ins and to mark the activities as completed...I didn't know how to go in and mark the check-in as completed so it looked like I didn't show up that day.

Unfortunately, I was unable to access and navigate through the program as I use an iPad.

...it was difficult for me to complete all the tasks. It took a lot of time \& energy which I don't seem to have. If the program could be scaled back some so the tasks didn't seem so daunting.

Participants overall seemed to desire an improved ease of use in order to successfully complete their assignments and fully engage in the online program.

\section{Discussion}

Participant perspectives provide an essential lens through which to evaluate E-health interventions. Five major themes emerged through our analysis of data provided by participants who completed the online pain self-management intervention.

Participants experienced the benefits of positive reframing, improved accountability, and feeling supported. Ideas for improving the program were described as a desire for personalizing, and improving ease of use.

Similar to other studies, the participants in the present study commonly reported that the online selfmanagement intervention taught them skills that enabled them to put a positive spin on their reaction to their chronic pain. Improving coping skills or decreasing "pain catastrophizing" have been noted as a benefit of several other online programs targeting self-management $[24,25,26]$. Throughout the data, participants described now having the tools to take a step back and reframe their negative thought patterns that were often associated with pain.

Akin to prior qualitative studies of people with chronic pain, the theme of feeling supported by others or not "being alone" in their suffering was important to participants [27]. Shared experiences, emotional support, and mutual understanding have been credited as key to success of similar interventions for people with chronic disease in group settings [27,28]. Fewer studies have reported this from online pain self-management, so it is worth noting that social support or feeling supported can be felt within online environments. It has been suggested that online forums offer a kind of support that cannot be achieved in face-to-face sessions, therefore, they offer novel types of support that may have unique benefits [29]. The ability to take control of one's disease and reduce inhibitions are two 
advantages noted to be afforded by online interactions [29]. Emotional and informational support are the types of support seen most commonly within online communities, and self-disclosure is more frequently seen online than within face-to-face support groups [30].

Prior studies have identified benefits of online self-management programs as improved accountability or improved awareness of ones' ability to choose positive actions and behaviors in living with and managing chronic pain [24,25,26]. People with chronic pain desire online programs that include information, activities and tools that will help them feel equipped to gain back control [26,31]. Our study participants often described that they appreciated receiving positive affirmations and reminders as part of the program and that this allowed them to check in with their progress. These actions served to remind them that they have the ability to improve their chronic pain experience through their own actions and gain some control. Increasing accountability meant that participants either increased their positive actions or took a step back in order to not further aggravate their pain.

Our study participants suggested increasing personalization of the program and improving ease of use in order for them to successfully complete the online program. Including subjective, individualized components to online programs is a common thread noted throughout the literature to meet participants' needs [24,25,26,31]. While the CPMP does offer daily tracking and activity scheduling that can be personalized, these features were also ones that seemed to cause the most technical difficulties for some participants. Offering an online diary component has proven beneficial for management of other chronic conditions [25]. Improving ease of use might be accomplished by providing more detailed orientation to the online program and offering other delivery methods such as iPad or hybrid learning options that include more personal contact along with virtual assistance.

The CPMP can be accessed from computers, tablets, and smartphones, and, thus, can be accessed in a variety of ways; however, training may be needed for participants to utilize all of the functions. At present, about two-thirds of the world use the Internet at least occasionally or own a smartphone, and the most dramatic rate increases have occurred in developing countries [32]. Mobile health technologies are believed to hold promise to reduce accessibility gaps in mental health services, yet, it is also recognized that efficacy research is in its infancy [33]. People are increasingly using the Internet for information and support, but more work is needed to tailor culturally-appropriate online pain resources and reduce disparities in access [17].

It was noted after analysis that the emerging themes matched the CPMP's four learning modules. "Thinking Clearly" aims to reframe to more positive thought patterns, and "Feeling Better" assists in identifying and removing negative emotional triggers. "Doing More" encourages accountability with goal-setting for activities. "Relating Better" addresses the need to feel supported within healthy relationships. That these themes emerged independently of any knowledge by one of the researchers on the program modules provides compelling evidence that the program was on target in achieving what it had intended.

Our qualitative descriptive approach guided by the theoretical framework of the IFSMT allowed us to identify specific variables unique to our participants' perspectives that can be explored in future intervention trials. For example, we recommend that a variety of accountability and support strategies be developed and tested for efficacy within online self-management interventions. For instance, health care providers can serve as collaborators, as the IFSMT dictates [22]. Providers can provide tangible support such as through in-person prompts, phone calls or text messages that encourage participants' progress towards goals. Family members can be enlisted to provide additional support to improve accountability. Online programs could be personalized by adding remedial training sessions for those who find computer programs daunting. Each new strategy can be tested for effects on proximal and distal outcomes as the IFSMT recommends. For example, adding family support might increase the short-term outcome of increasing physical exercise, which then might increase the desired long-term outcome of reduced health care utilization.

Limitations of the study include the inability to question or clarify participant remarks further. The data provided was collected online and those who prefer to use verbal rather than written communications may not have fully participated. Focus groups, interviews, or online chat sessions might have allowed for more detailed input from participants. The sample was restricted to U.S. residents so may not apply to other cultures or settings. While some participants were recruited from clinical settings, many self-referred from the Internet so may not be representative of all people with chronic pain. Respondents were predominantly Caucasian and educated, like most other studies of online pain management [19], limiting generalizability. Yet, strengths of the study included the 
number and depth of participants' responses, and that participants' were able to maintain anonymity, which has been found to be an advantage of online research [35]. The privacy provided may have allowed more honest disclosures and freedom of expression.

Our previously published RCT provided quantitative evidence of both the efficacy and the limits of the CPMP [3], and the participants' words presented here extend, amplify, and clarify that evidence. The original published RCT included a summative program evaluation using an adapted version of the IBM Computer Usability Satisfaction Questionnaire. Seven Likert-style scale items were presented to rate satisfaction with the program's usability and usefulness, and the mean value of the combined evaluation items was interpreted to be high [3]. Yet from those data, we gleaned little insight about what participants might need to maximize the programs' usefulness and engage more vigorously, nor could we fully appreciate individual benefits. It is apparent from the deeper inquiry presented here that gains can be made using E-health for chronic pain, and also that more development is needed to fully support participants in their efforts to self-manage symptoms.

\section{Conclusion}

The identified themes provide specific areas that can be targeted for developing online interventions that can improve pain management and quality of life. Participants' insights regarding perceived benefits and challenges can assist health care providers in understanding how such programs may assist in chronic disease self-management for pain and a multitude of health problems.

\section{References}

[1] Institute of Medicine Committee on Advancing Pain, Research, Care, and Education, "Relieving Pain in America: A Blueprint for Transforming Prevention, Care, Education, and Research", National Academies Press, Washington, D.C., 2011.

[2] R. Knoerl, E.M. Lavoie Smith, and J. Weisberg, "Chronic Pain and Cognitive Behavioral Therapy: An Integrative Review", Western Journal of Nursing Research, 2016, 38(5), pp. 596-628. doi: 10.1177/0193945915615869

[3] M. Wilson, J.M. Roll, C. Corbett, and C. BarbosaLeiker, "Empowering Patients with Persistent Pain using an Internet-based Self-Management Program", Pain Management Nursing, 2015, 16(4), pp. 503-14. doi: 10.1016/j.pmn.2014.09.009; PMID:26088940
[4] R.L. Nahin, "Estimates of Pain Prevalence and Severity in Adults: United States, 2012", Journal of Pain, 2015, 16(8), pp. 769-780.

[5] J. Kennedy, J.M. Roll, T. Schraudner, S. Murphy, and S. McPherson, "Prevalence of Persistent Pain in the U.S. Adult Population: New Data from the 2010 National Health Interview Survey", Journal of Pain, 2014, 15(10), pp. 979984.

[6] D.D. Macea, K. Gajos, Y.A. Calil, and F. Fregni, "The Efficacy of Web-based Cognitive Behavioral Interventions for Chronic Pain: A Systematic and Meta-analysis", The Journal of Pain, 2010, 11(10), pp. 917-929. doi:10.1016/j/jpain.2010.06.005

[7] C. Eccleston, "Can 'Ehealth' Technology Deliver on its Promise of Pain Management for All?", Pain, 2011, 152(8), pp. 1701-1702.

[8] Centers for Disease Control and Prevention (CDC), "Prescription Drug Overdose in the United States: Fact sheet July 3, 2014", http://www.cdc.gov/drugoverdose /data/prescribing.html, Accessed March 22, 2016.

[9] L. Manchikanti, S. Abdi, S. Atluri, C.C Balog, R.M. Benyamin, M.V. Boswell,...American Society of Interventional Pain Physicians, "American Society of Interventional Pain Physicians (ASIPP) Guidelines for Responsible Opioid Prescribing in Chronic Non-cancer Pain: Part I--Evidence Assessment", Pain Physician, 2012, 15(3 Suppl), pp. S1-65.

[10] E.O. Dumas, and G.M. Pollack, "Opioid Tolerance Development: A Pharmacokinetic/Pharmacodynamic Perspective", The AAPS Journal, 2008, 10(4), pp. 537-551. doi:10.1208/s12248-008-9056-1

[11] Department of Health and Human Services (DHHS). "National Pain Strategy", March 18, 2016, https://iprcc.nih.gov/National_Pain_Strategy/NPS_Main.ht $\mathrm{m}$, Accessed March 22, 2016.

[12] R. Chou, G. Fanciullo, P. Fine, J. Adler, J. Ballantyne, P. Davies,...American Pain Society-American Academy of Pain Medicine Opioids Guidelines Panel, "Clinical Guidelines for the Use of Chronic Opioid Therapy in Chronic Noncancer Pain", Journal of Pain, 2009, 10(2), pp. 113-30.

[13] L. Manchikanti, H. Ailinani, D. Koyyalagunta, S. Datta, V. Singh, I. Eriator,... P.A. Christo, "A Systematic Review of Randomized Trials of Long-term Opioid Management for Chronic Non-cancer Pain", Pain Physician, 2011, 14, pp. 91-121.

[14] R. Hallinan, M. Osborn, M. Cohen, M. Dobbin, and A. Wodak, "Increasing the Benefits and Reducing the Harms of Prescription Opioid Analgesics", Drug and Alcohol Review, 2011, 30, pp. 315-323. 
[15] K. Lorig, P. Ritter, D. Laurent, and K. Plant, "The Internet-based Arthritis Self-management Program: A Oneyear Randomized Trial for Patients with Arthritis or Fibromyalgia", Arthritis \& Rheumatism, 2008, 59(7), pp. 1009-1017.

[16] Institute for Clinical Systems Improvement (ICSI), "Pain, Chronic; Assessment and Management of (Guideline)", 2011, http://www.icsi.org/guidelines, Accessed March 20, 2016.

[17] S. Ziebland, M. Lavie-Ajayi, and G. Lucius-Hoene, "The Role of the Internet for People with Chronic Pain: Examples from the DIPEx International Project", British Journal of Pain, 2015, 9(1), pp. 62-64.

[18] L. Ruehlman, P. Karoly, and C. Enders, "A Randomized Controlled Evaluation of an Online Chronic Pain Self-Management Program", Pain, 2011, doi:10.1016/j.pain.2011.10.025

[19] J.L. Bender, A. Radhakrishnan, C. Diorio, M. Englesakis, and A.R. Jadad, "Can Pain be Managed through the Internet? A Systematic Review of Randomized Controlled Trials", Pain, 2011, 152, pp. 1740-50.

[20] M. Sandelowski, "What's in a Name? Qualitative Description Revisited," Research in Nursing \& Health, 2010, 33, pp. 77-84.

[21] M. Sandelowski, "Focus on Research Methods Whatever Happened to Qualitative Description?" Research in Nursing \& Health, 2000, 23, pp. 334-340.

[22] P. Ryan, and K. Sawin, "The Individual and Family Self-Management Theory: Background and Perspectives on Context, Process, and Outcomes", Nursing Outlook, 2009, 57, pp. 217-225.

[23] M. Schreier. Qualitative Content Analysis in Practice, Sage, Thousand Oaks, CA, 2012.

[24] O.B. Kristjansdottir, E.A. Fors, A. Finset, S. Dulmen, S.H. Wigers, and H. Eide, "Written Online Situational Feedback via Mobile Phone to Support Self-management of Chronic Widespread Pain: A Usability Study of a Webbased Intervention", BioMed Central Musculoskeletal Disorders, 2011, 12(51), http://www. biomedcentral.com/ 1471-2474/12/51, Accessed June 1. 2016.

[25] V. Kruijssen, A. Staa, J. Dwarswaard, J. Veen, B. Mennema, and S. Adams, "Use of Online SelfManagement Diaries in Asthma and COPD: A Qualitative Study of Subjects' and Professionals' Perceptions and Behaviors", Respiratory Care, 2015 60(8), pp. 1146-1156. doi: 10.4187/respcare.03795

[26] K. Trudea, L. Pujol, P. DasMahapatra, R. Wall, R. Black, and K. Zacharoff, "A Randomized Controlled Trial of an Online Self-management Program for Adults with Arthritis Pain", Journal of Behavioral Medicine, 2015, 38, pp. 483-496. doi: 10.1007/s10865-015-9622-9
[27] L.N. Andersen, M. Kohberg, L.G. Herborg, K. Søgaard, and K.K. Roessler, ""Here We're All in the Same Boat"-- A Qualitative Study of Group Based Rehabilitation for Sick-listed Citizens with Chronic Pain", Scandinavian Journal of Psychology, 2014, 55(4), pp. 333-42, doi: 10.1111/sjop.12121.

[28] J. Barlow, R. Edwards, and A. Turner, "The Experience of Attending a Lay-led, Chronic Disease Selfmanagement Programme from the Perspective of Participants with Multiple Sclerosis", Psychological Health, 2009, 24(10), pp. 1167-80, doi: $10.1080 / 08870440802040277$

[29] A. Broom, "Virtually He@lthy: The Impact of Internet Use on Disease Experience and the Doctor-Patient Relationship", Qualitative Health Research, 2005, 15(3), pp. 325-45.

[30] K. M. Griffiths, A.L. Calear, M. Banfield, and A. Tam, "Systematic Review on Internet Support Groups (ISGs) and Depression (2): What Is Known About Depression ISGs?", Journal of Medical Internet Research, 2009, 11(3), e41, doi: 10.2196/jmir. 1303

[31] M. Nielsen, G. Jull, and P.W. Hodges, "Information Needs of People with Low Back Pain for an Online Resource: A Qualitative Study of Consumer Views", Disability and Rehabilitation, 2014, 36(13), pp. 1085-1091, doi: 10.3109/09638288.2013.829532

[32] Pew Research Center, "Smartphone Ownership and Internet Usage Continues to Climb in Emerging Economies", http://www.pewglobal.org/2016/02/22/smart phone-ownership-and-internet-usage-continues-to-climbin-emerging-economies/, Accessed August 21, 2016.

[33] E. Anthes, "Mental Health - There's an App for That", Nature: International Weekly Journal of Science, April 6, 2016, http://www.nature.com/news/mental-health-there-san-app-for-that-1.19694, Accessed August 21, 2016.

[34] R. Hamilton R., and B. Bowers, "Internet recruitment and e-mail interviews in qualitative studies", Qualitative Health Research, 2006 16, pp. 821, doi: $10.1177 / 1049732306287599$

\section{Acknowledgements}

The data collection for this research was supported in part by the Washington State Life Sciences Discovery Fund (grant LSDF 08-02, John Roll, PI). The authors acknowledge Dr. Linda Ruehlman and Dr. Paul Karoly, creators of the Chronic Pain Management Program, for assistance in enrolling participants and sharing program activity data. 